\title{
miR-665 is downregulated in glioma and inhibits tumor cell proliferation, migration and invasion by targeting high mobility group box 1
}

\author{
HAO SHEN, LING XU, CHUNYUE YOU, HUAIBO TANG, HAITAO WU, YONG ZHANG and MINGXIANG XIE
}

Department of Neurosurgery, Affiliated Hospital of Zunyi Medical University, Zunyi, Guizhou 563003, P.R. China

Received September 25, 2019; Accepted October 22, 2020

DOI: $10.3892 / \mathrm{ol} .2020 .12417$

\begin{abstract}
Glioma is the most common brain tumor in adults. microRNAs (miRNAs/miRs) play an essential role in tumor development and progression. The present study aimed to investigate the potential clinical significance and function of miR-665 in glioma. Reverse transcription-quantitative PCR analysis was used to detect the expression of miR-665 in glioma tissues and cells. Survival curves were constructed using the Kaplan-Meier method. Cox regression analysis was performed to investigate the prognostic significance of miR-665. Cell Counting Kit-8 and Transwell assays were used to evaluate the role of miR-665 in glioma. Bioinformatics analysis and Dual-luciferase reporter assays were used to predict the putative direct targets of miR-665. Western blotting was used to evaluate the activity of the Wnt/ $\beta$-catenin pathway. The relative expression of miR-665 was decreased in glioma tissues and cells and this downregulation was significantly associated with the Karnofsky performance scale score and World Health Organisation grade. Patients with glioma with low miR-665 expression had a shorter overall survival time compared with the high expression group. Besides, overexpression of miR-665 suppressed the proliferation, migration and invasion of glioma cells, while knockdown of miR-665 promoted these cellular behaviors. High mobility group box (HMGB)1 was a direct target of miR-665. It was also demonstrated that miR-665 may suppress glioma progression by targeting HMGB1 and inhibiting the Wnt/ $\beta$-catenin pathway. Taken together, these data suggested that miR-665 may have a tumor suppressor role in glioma by targeting HMGB1. Therefore, miR-665 may be a novel prognostic biomarker and the miR-665/HMGB1 axis may be a novel therapeutic target for the treatment of glioma.
\end{abstract}

Correspondence to: Professor Mingxiang Xie, Department of Neurosurgery, Affiliated Hospital of Zunyi Medical University, 149 Dalian Road, Zunyi, Guizhou 563003, P.R. China

E-mail: mingxiangxie@163.com

Key words: microRNA-665, glioma, prognosis, progression, high mobility group box 1

\section{Introduction}

Glioma is the most common type of primary brain tumor that originates from glial cells, with an overall incidence of $\sim 5 / 100,000$ individuals per year worldwide $(1,2)$. According to the World Health Organization (WHO) grading criteria, gliomas are categorized into low-grade gliomas (grade I and II) and high-grade gliomas (grade III and IV) (1). Glioma exhibits several malignant characteristics, such as strong invasiveness and a high recurrence rate $(3,4)$. Despite considerable advances in surgery, radiotherapy and chemotherapy, the median survival time of patients with glioma has only marginally improved (5). In particular, grade IV glioma, also known as glioblastoma, is the most aggressive form of glioma with a median survival of 12-15 months $(6,7)$. Therefore, the identification of novel cancer-associated biomarkers may be beneficial for improving the prognosis of patients with glioma.

microRNAs (miRNAs/miRs) are a class of highly conserved small non-coding RNAs that post-transcriptionally modulate the expression of mRNAs by binding to their 3'-untranslated region (UTR) (8). Studies have shown that miRNAs play a crucial role in various biological processes, including cell proliferation, differentiation, apoptosis and migration $(9,10)$. Aberrant miRNA expression is involved in the progression of various diseases, including cancer $(11,12)$. To date, several miRNAs have been identified to function as oncogenes or suppressor genes in cancer development, such as miR-21 in colorectal cancer (13) and miR-338 in glioma (14), which suggests that they are potential therapeutic targets and prognostic biomarkers (15). Abnormal miR-665 expression has been reported in multiple human diseases, including cancer (16-18). For example, a recent study identified several glioma-associated miRNAs, including miR-665 (19). To the best of our knowledge, the clinical significance and functional role of miR-665 in glioma have not been investigated. High mobility group box 1 (HMGB1) is upregulated in tumor tissues or cancer cell lines and contributes to the development of a variety of cancer types, such as gastric and colorectal cancer and hepatocellular carcinoma (20-22). Previous studies have demonstrated an association between HMGB1 and the $\mathrm{Wnt} / \beta$-catenin pathway in esophageal squamous cell carcinoma (23) and colorectal carcinoma (24). Accordingly, it was hypothesized the miR-665/HMGB1 axis may be 
involved in the progression of glioma through regulating the Wnt/ $\beta$-catenin pathway.

The present study aimed to examine the expression of miR-665 in glioma using reverse transcription quantitative PCR (RT-q)PCR and explore its clinical significance using Kaplan-Meier method. The functional role of miR-665 was also investigated using cellular experiments in vitro, demonstrating the role of the miR-665/HMGB1 axis in regulating the progression of glioma through the $\mathrm{Wnt} / \beta$-catenin pathway.

\section{Materials and methods}

Patients and tissue specimens. The present study was approved by The Ethics Committee of Affiliated Hospital of Zunyi Medical University (approval no. SYXK Qin 2018-0006), and written informed consent was obtained from the patients with glioma. Paired fresh glioma tissue specimens and adjacent non-cancerous tissue specimens (3-cm away from glioma) were obtained from a total of 113 glioma patients who underwent resection at Affiliated Hospital of Zunyi Medical University between March 2010 and May 2014. Inclusion criteria were as follows: i) patients had not received anticancer treatment before surgical resection; ii) complete clinicopathological characteristic information; and iii) patients had no other diseases. All tissue specimens were made anonymous, confirmed by experienced pathologists independently and immediately snap-frozen and stored in liquid nitrogen until RNA extraction. Detailed clinicopathological characteristics of all the patients, including age, sex, tumor size, Karnofsky performance scale (KPS) and WHO grade $(25,26)$, were obtained. For prognostic analyses, the 5-year overall survival information of patients was collected after surgery and recorded by telephone every 3 months during the first 2 years, every 6 months during the next 2 years and one year thereafter until death events occurred or the cut-off follow-up date. In the clinical analysis, glioma patients were divided into low miR-665 expression group and high miR-665 expression group according to the mean miR-665 expression level (0.2168) in glioma tissues.

Cell culture and transfection. The human glioma cell lines (U251MG, A172, LN18 and T98G) were purchased from The Cell Bank of Type Culture Collection of the Chinese Academy of Sciences. All glioma cells were incubated in DMEM (Gibco; Thermo Scientific, Inc.) containing 10\% FBS (Gibco; Thermo Fisher Scientific, Inc.). Normal human astrocytes (NHAs; cat. no. CC-2565) were purchased from Lonza Group, Ltd. and cultured in astrocyte growth media (Lonza Group, Ltd.) supplemented with 10\% FBS (Thermo Fisher Scientific, Inc.). All cells were incubated at $37^{\circ} \mathrm{C}$ in a humidified atmosphere containing $5 \% \mathrm{CO}_{2}$.

The miR-665 mimic (5'-ACCAGGAGGCUGAGGCCC CU-3'), miR-665 inhibitor (5'-AGGGGCCUCAGCCUCCUG GU-3'), mimic non-targeting negative control (NC; 5'-UUC UCCGAACGUGUCACGUTT-3'), inhibitor non-targeting NC (5'-CAGUACUUUUGUGUAGUACAA-3'), pcDNA3.1 (negative control for pcDNA3.1-HMGBI) and pcDNA3.1-HMGBI were synthesized by Shanghai GenePharma Co., Ltd. For cell transfection, $2 \times 10^{5}$ glioma cells were seeded into 6-well plates, and miR-665 mimic, mimic NC, miR-665 inhibitor, inhibitor NC, pcDNA3.1 or pcDNA3.1-HMGB1 were transfected into
A172 and T98G cells using Lipofectamine ${ }^{\circledR} 3000$ reagent (Invitrogen; Thermo Fisher Scientific, Inc.) according to the manufacturer's protocols. After transfection for $48 \mathrm{~h}$, the transfection efficiency was confirmed by RT-qPCR and transfected cells were collected for further analysis.

$R N A$ extraction and reverse transcription quantitative PCR $(R T-q) P C R$. Total RNA was extracted from glioma tissues and normal cells and glioma cells using TRIzol ${ }^{\circledR}$ reagent (Invitrogen; Thermo Fisher Scientific, Inc.) following the manufacturer's instructions. Afterwards, the RNA was reverse transcribed to cDNA using the PrimeScript ${ }^{\mathrm{TM}}$ RT Reagent kit (Takara Biotechnology Co., Ltd.) according to the manufacturer's protocol. The miR-665 reverse transcription primer was 5'-CTCAACTGGTGTCGTGGAGTCGGCAATTCA GTTGAGAGGGGC-3'. Then, qPCR was performed using the SYBR-Green PCR kit (Thermo Fisher Scientific, Inc.) on an ABI 7900 thermocycler (Applied Biosystems; Thermo Fisher Scientific, Inc.). The thermocycling conditions for RT-qPCR were as follows: Initial denaturation at $95^{\circ} \mathrm{C}$ for $3 \mathrm{~min}$, followed by 35 cycles of $95^{\circ} \mathrm{C}$ for $30 \mathrm{sec}, 60^{\circ} \mathrm{C}$ for $30 \mathrm{sec}$ and $72^{\circ} \mathrm{C}$ for $30 \mathrm{sec}$. The primers used were as follows: miR-665, forward: 5'-GCCGAGACCAGGAGGCTGAG-3' and reverse: 5'-CTC AACTGGTGTCGTGGA-3'; U6, forward: 5'-CTCGCTTCG GCAGCACA-3' and reverse: 5'-AACGCTTCACGAATTTGC GT-3'; HMGB1, forward: 5'-TCCAGCAGTTTGTTGGGGTT-3' and reverse: 5'-AACAAGGGCCTAAGCGAGTC-3'; $\beta$-actin, forward: 5'-AAGACGTACTCAGGCCATGTCC-3' and reverse: 5'-GACCCAAATGTCGCAGTCAG-3'. Relative miR-665 and $H M G B 1$ mRNA expression levels were analyzed using the $2^{-\Delta \Delta C q}$ method (27) and normalized to U6 and $\beta$-actin, respectively.

Cell proliferation assay. The cell proliferation rate was analyzed using a Cell Counting Kit (CCK)-8 (Beyotime Institute of Biotechnology). A total of $2 \times 10^{3}$ transfected cells/well were seeded into 96-well plates and incubated for $0,24,48$ and $72 \mathrm{~h}$ at $37^{\circ} \mathrm{C}$ before the addition of $10 \mu \mathrm{l} \mathrm{CCK}-8$ reagent. Cells were incubated for a further $2 \mathrm{~h}$, and optical density of each well was measured at a wavelength of $450 \mathrm{~nm}$ using a microplate reader (Bio-Rad Laboratories, Inc.).

Cell migration and invasion assay. The migration and invasion capabilities of glioma cells were evaluated using Transwell inserts ( $8-\mu \mathrm{m}$ pores; BD Biosciences). In the invasion assays, the membranes were pre-coated with Matrigel (BD Biosciences) at $37^{\circ} \mathrm{C}$ for $1 \mathrm{~h}$, while a non-Matrigel insert was used for the migration assays. After transfection, $5 \times 10^{4}$ transfected cells were re-suspended in FBS-free medium and placed into the upper chambers, while the medium with $10 \%$ FBS was added into the lower chambers. After $24 \mathrm{~h}$, migratory and invasive cells in the lower membrane were fixed with $4 \%$ paraformaldehyde at room temperature for $20 \mathrm{~min}$ and stained with $0.1 \%$ crystal violet at $37^{\circ} \mathrm{C}$ for $15 \mathrm{~min}$. Stained cells were counted from five random fields under a light microscope (magnification, x200; Olympus Corporation).

Bioinformatics analysis and Dual-luciferase reporter assay. Bioinformatics analyses were performed using TargetScan (www.targetscan.org) and miRWalk (mirwalk.umm. uni-heidelberg.de/) to predict the potential target genes of 

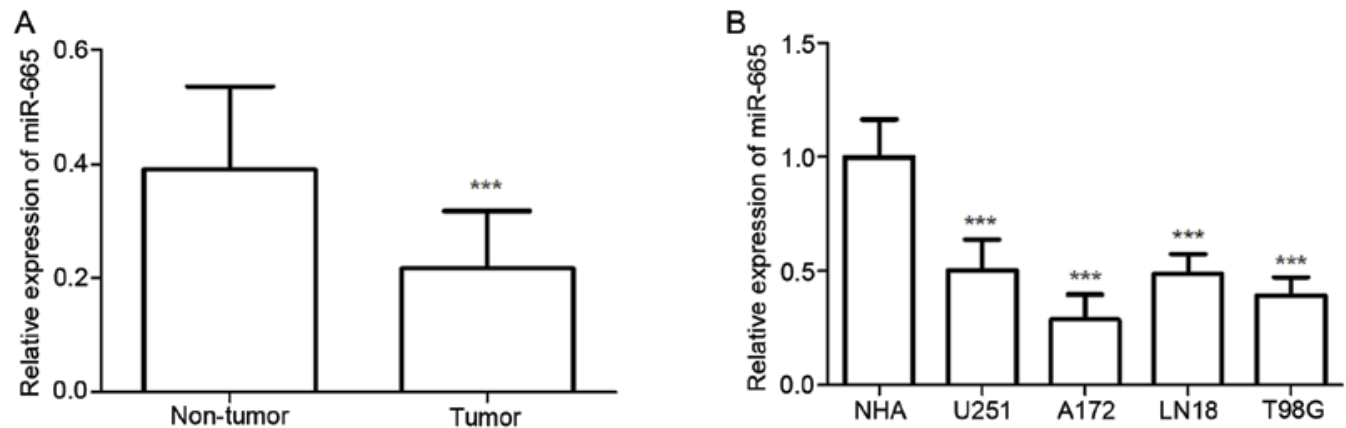

Figure 1. Expression of miR-665 in glioma tissues and cell lines measured using reverse transcription-quantitative PCR. (A) miR-665 expression was significantly lower in glioma tissues compared with adjacent normal tissues. (B) Relative expression of miR-665 was downregulated in glioma cell lines (U251MG, A172, LN18 and T98G) compared with NHAs. ${ }^{* * *}$ P $<0.001$ vs. non-tumor or NHA. miR, microRNA; NHA, normal human astrocytes.

miR-665. The predicted wild-type (wt) or mutant (mut) 3'-UTR of $H M G B 1$ was amplified and cloned into a pmirGLO plasmid (Promega Corporation), generating the plasmids $H M G B 1$ 3'-UTR-wt and HMGB1 3'-UTR-mut, respectively. Then, the generated vectors were cotransfected with miR-665 mimic, mimic NC, miR-665 inhibitor or inhibitor NC into A172 cells using Lipofectamine ${ }^{\circledR} 3000$ reagent as aforementioned. After $48 \mathrm{~h}$ of transfection, the firefly luciferase activity was determined using the Dual-Luciferase Reporter Assay system (Promega Corporation) by normalizing to Renilla luciferase activity.

Western blotting. Transfected A172 cells were cultured and prepared in 6-well plates overnight. The cells were then harvested and washed twice with PBS and lysed with RIPA lysis buffer (Beyotime Institute of Biotechnology) to extract total protein. The protein concentration was determined using a BCA protein assay kit (Beyotime Institute of Biotechnology). For western blot analysis, proteins were separated via $10 \%$ SDS-PAGE and then blotted onto nitrocellulose membranes. The membranes were blocked in $5 \%$ milk for $1 \mathrm{~h}$ at room temperature and were then incubated with primary antibodies against $H M G B 1$ (cat. no. ab18256; 1:1,000 dilution), $\beta$-catenin (cat. no. ab32572; $1: 1,000$ dilution), cyclin D1 (cat. no. ab16663; 1:2,000 dilution), C-myc (cat.no. ab32072; 1:1,000 dilution) (all from Abcam) and $\beta$-actin (cat. no. sc-47778; 1:1,000 dilution; Santa Cruz Biotechnology, Inc.) overnight at $4^{\circ} \mathrm{C}$. After that, the membranes were incubated with corresponding secondary antibody goat anti-rabbit HRP (cat. no. ab205718; 1:5,000 dilution; Abcam) or goat anti-mouse HRP (cat. no. ab6789; 1:5,000 dilution; Abcam) for $1 \mathrm{~h}$ at room temperature. The signals were visualized using an electrochemiluminescence kit (GE Healthcare) and protein levels were quantified by densitometry using ImageJ version (1.42 software; National Institutes of Health).

Statistical analysis. All experiments were repeated at least three times and data are presented as the mean \pm SD, unless otherwise shown. The statistical analyses were performed using SPSS 20.0 (IMB Corp.) and GraphPad Prism 5 software (GraphPad Software Inc.). $\chi^{2}$ tests were used to analyze the association between miR-665 expression and clinical characteristics of patients with glioma. Paired Student's t-tests were used to compare differences between tumor and adjacent non-tumor tissues and one-way ANOVA followed by a Tukey's post hoc test was performed to analyze the difference among three or more groups. Kaplan-Meier curves with log-rank tests and Cox regression analysis were performed to investigate the prognostic significance of miR-665. $\mathrm{P}<0.05$ was considered to indicate a statistically significant difference.

\section{Results}

Decreased miR-665 expression in glioma tissues and cells. The expression of miR-665 in both glioma tissues and cell lines was detected using RT-qPCR. The results showed glioma tissues exhibited significantly decreased relative miR-665 expression compared with normal tissues ( $\mathrm{P}<0.001$; Fig. 1A). As demonstrated in Fig. 1B, miR-665 expression was significantly decreased in glioma cells, including U251MG, A172, LN18 and T98G cells, compared with expression in NHA cells (all $\mathrm{P}<0.001$ ). These results suggested that miR-665 may be a tumor suppressor in glioma. Among these glioma cells, A172 and T98G exhibited the lowest miR-665 expression levels, therefore these cell lines were selected for further experiments.

Association between miR-665 expression and clinicopathological characteristics and prognosis of patients with glioma. Patients with glioma were classified into low $(n=58)$ and high $(n=55)$ miR-665 expression groups based on the mean miR-665 expression level in glioma tissues. The present study demonstrated that low miR-665 expression was significantly associated with KPS $(\mathrm{P}=0.031)$ and WHO grade $(\mathrm{P}=0.006)$ in patients with glioma; however, no significant association was observed between miR-665 expression and other parameters, including age, sex and tumor size (Table I). These results suggested that miR-665 expression may be involved in the development of glioma.

Furthermore, a Kaplan-Meier curve was constructed to analyze 5-year follow-up data (the average follow-up time was 39.36 months), which suggested that patients with a low expression level of miR-665 had a significantly shorter overall survival time compared with the high expression group ( $\mathrm{P}=0.008$; Fig. 2). Furthermore, multivariate Cox regression analysis revealed that the expression level of miR-665 and WHO grade were independent indicators for overall survival in patients with glioma $(\mathrm{P}<0.05$; Table II). Overall, these 
Table I. Association of high $(\mathrm{n}=55)$ and low $(\mathrm{n}=58)$ microRNA-665 with the clinicopathological features of 113 patients with glioma.

\begin{tabular}{|c|c|c|c|c|}
\hline \multirow[b]{2}{*}{ Features } & \multirow[b]{2}{*}{ No. of cases } & \multicolumn{2}{|c|}{$\begin{array}{c}\text { miR-665 } \\
\text { expression, } \mathrm{n}\end{array}$} & \multirow[b]{2}{*}{ P-value } \\
\hline & & Low & High & \\
\hline Age, years & & & & 0.383 \\
\hline$\leq 50$ & 52 & 29 & 23 & \\
\hline$>50$ & 61 & 29 & 32 & \\
\hline Sex & & & & 0.482 \\
\hline Female & 49 & 27 & 22 & \\
\hline Male & 64 & 31 & 33 & \\
\hline Tumor size, $\mathrm{cm}$ & & & & 0.636 \\
\hline$\leq 5.0$ & 57 & 28 & 29 & \\
\hline$>5.0$ & 56 & 30 & 26 & \\
\hline KPS & & & & 0.031 \\
\hline$\leq 80$ & 57 & 35 & 22 & \\
\hline$>80$ & 56 & 23 & 33 & \\
\hline WHO grade & & & & 0.006 \\
\hline I-II & 59 & 23 & 36 & \\
\hline III-IV & 54 & 35 & 19 & \\
\hline
\end{tabular}

WHO, World Health Organization; KPS, Karnofsky performance scale.

results indicated that decreased miR-665 may be a prognostic biomarker in glioma.

miR-665 suppresses proliferation, migration and invasion of glioma cells. To further investigate the functional relevance of miR-665 in glioma, A172 and T98G cells were transfected with miR-665 mimic, mimic NC, miR-665 inhibitor or inhibitor NC and were analyzed using RT-qPCR. The relative expression of miR-665 was significantly increased in glioma cells following transfection with miR-665 mimic, and was significantly decreased following transfection with the miR-665 inhibitor, compared with untreated cells $(\mathrm{P}<0.001$; Fig. $3 \mathrm{~A})$. The mimic $\mathrm{NC}$ and inhibitor NC group were not significantly different compared with the untreated group, therefore untreated cells were used as control comparisons. Cell proliferation was examined using CCK-8 assays, which revealed that overexpression of miR-665 significantly inhibited glioma cell proliferation, whereas knockdown of miR-665 significantly promoted cell proliferation, compared with untreated cells $(\mathrm{P}<0.05$; Fig. $3 \mathrm{~B})$. Transwell migration and invasion assays revealed that upregulation of miR-665 significantly suppressed the migration and invasion of glioma cells, while inhibition of miR-665 attenuated these abilities, compared with untreated cells $(\mathrm{P}<0.01$ or $\mathrm{P}<0.001$; Fig. 4A-D). These results indicated that miR-665 may exert an tumor suppressive role in glioma through suppressing tumor cell proliferation, migration, and invasion.

miR-665 directly targets HMGB1 in A172 cells. According to the bioinformatics prediction, the 3'-UTR region of the HMGB1

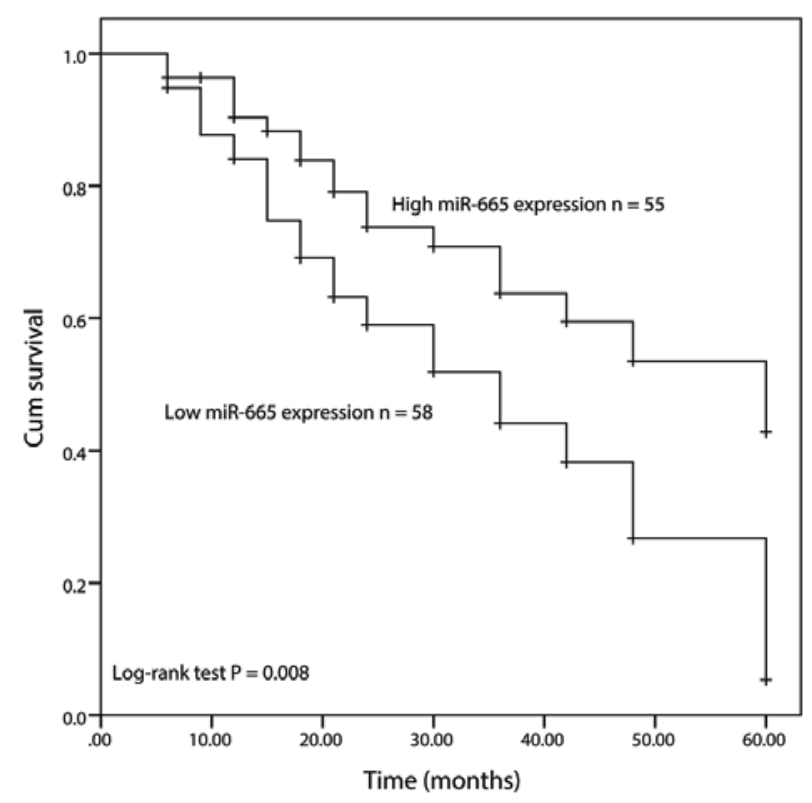

Figure 2. Kaplan-Meier survival analysis of the association between miR-665 expression and overall survival time of patients with glioma. miR, microRNA; Cum, cumulative.

gene was complementary with the seed sequences of miR-665 (Fig. 5A). Firstly, HMGB1 mRNA and protein expression levels were quantified using RT-qPCR and western blotting, respectively. The results showed that miR-665-overexpression inhibited both HMGB1 mRNA and protein levels, while knockdown of miR-665 promoted both HMGB1 mRNA and protein levels in A172 cells, compared with the untreated group $(\mathrm{P}<0.001$; Fig. 5B and C). Subsequently, the luciferase reporter assay results revealed that enhanced expression of miR-665 significantly inhibited the luciferase activity of the HMGB1 3'-UTR wt, while downregulation of miR-665 promoted the luciferase activity of the HMGB1 3'-UTR wt, compared with the untreated group $(\mathrm{P}<0.05$; Fig. 5D). Meanwhile, no significant changes were observed in the HMGB1 3'-UTR mut. These results revealed that miR-665 could directly target $H M G B 1$ in A172 cells.

Effects of miR-665 and HMGB1 on the activity of the Wnt/ $\beta$-catenin signaling pathway. A previous study reported that miR-665 inhibits tumorigenesis by directly targeting $H M G B 1$ and inactivating the $\mathrm{Wnt} / \beta$-catenin pathway in retinoblastoma (28). Thus, the present study investigated whether miR-665 and $H M G B 1$ expression could affect the Wnt/ $\beta$-catenin signaling pathway in A172 cells. First, the transfection efficiency was measured using RT-qPCR and the results are shown in Fig. $\mathrm{S} 1$ ( $\mathrm{P}<0.001$ vs. untreated cells). The expression levels of the important molecules in the Wnt $/ \beta$-catenin signaling pathway, including $\beta$-catenin, cyclin D1 and C-myc (29), were measured in A172 cells with the $\beta$-actin as internal reference following the altered expression of miR-665 and HMGB1. As presented in Fig. 6, overexpression of miR-665 significantly inhibited the protein levels of $\beta$-catenin, cyclin D1 and C-myc, while recovery of $H M G B 1$ expression partially abrogated the inhibition of $\beta$-catenin, cyclin D1 and C-myc protein levels 
Table II. Multivariate Cox regression analysis for clinicopathological variables of patients with glioma.

\begin{tabular}{lccr}
\hline & \multicolumn{2}{c}{ Multivariate analysis } \\
\cline { 2 - 4 } Variables & HR & $95 \%$ CI & P-value \\
\hline miR-665, low vs. high expression & 1.878 & $1.058-3.333$ & 0.031 \\
Age, $\leq 50$ vs. $>50$ years & 0.801 & $0.452-1.418$ & 0.447 \\
Sex, female vs. male & 0.726 & $0.358-1.472$ & 0.375 \\
Tumor size, $>5.0$ vs. $\leq 5.0 \mathrm{~cm}$ & 1.927 & $0.934-3.975$ & 0.076 \\
KPS, $>80$ vs. $\leq 80$ & 0.564 & $0.317-1.006$ & 0.052 \\
WHO grade, III-IV vs. I-II & 1.784 & $1.013-3.144$ & 0.045 \\
\hline
\end{tabular}

WHO, World Health Organization; KPS, Karnofsky performance scale; HR, hazard ratio; CI, confidence interval.
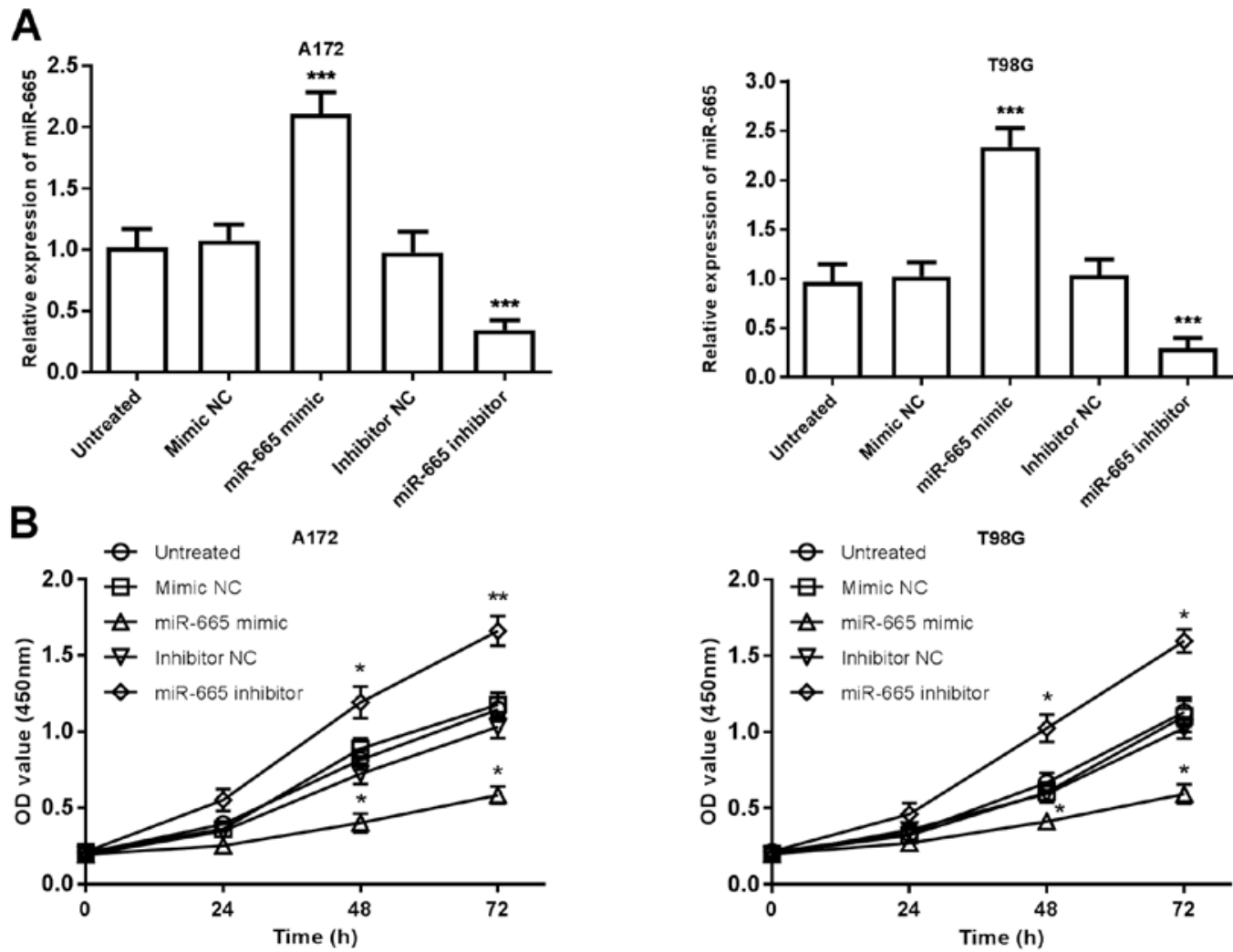

Figure 3. Effects of miR-665 on the proliferation of A172 and T98G cells. (A) Transfection efficiency was confirmed using reverse transcription-quantitative PCR in cells following transfection with miR-665 mimic, miR-665 inhibitor or respective NCs. (B) Overexpression of miR-665 suppressed cell proliferation, while knockdown of miR-665 promoted cell proliferation, compared with untreated cells. ${ }^{*} \mathrm{P}<0.05,{ }^{* * *} \mathrm{P}<0.01,{ }^{* * * * *} \mathrm{P}<0.001$ vs. untreated control. miR, microRNA; $\mathrm{NC}$, negative control; OD, optical density.

caused by miR-665 upregulation, compared with untreated group $(\mathrm{P}<0.05)$. These results suggested that miR-665 deactivated the Wnt/ $\beta$-catenin pathway in A172 cells through the negative mediation of $H M G B 1$.

\section{Discussion}

Glioma, a malignant brain tumor, is one of the most common malignancies with high mortality rate in China, and there were $\sim 61,000$ brain cancer-associated deaths in 2015 (30). To find more effective treatments for glioma, there is a need to identify valuable cancer-related biomarkers. In the present study, it was observed that miR-665 expression was significantly decreased in glioma tissues and cell lines compared with normal tissues and NHAs, and miR-665 expression was associated with KPS and WHO grade. Low expression levels of miR-665 was associated with poor prognosis of patients with glioma. Besides, overexpression of miR-665 significantly inhibited proliferation, migration and invasion of glioma cells, while knockdown of miR-665 promoted these cellular behaviors by targeting HMGB1 through regulating the Wnt/ $\beta$-catenin pathway.

In recent years, growing evidence has demonstrated that miRNAs function as diagnostic and/or prognostic biomarkers 
A

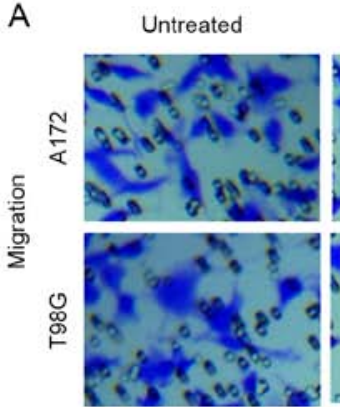

Mimic NC

miR-665 mimic

Inhibitor NC miR-665 inhibitor

B
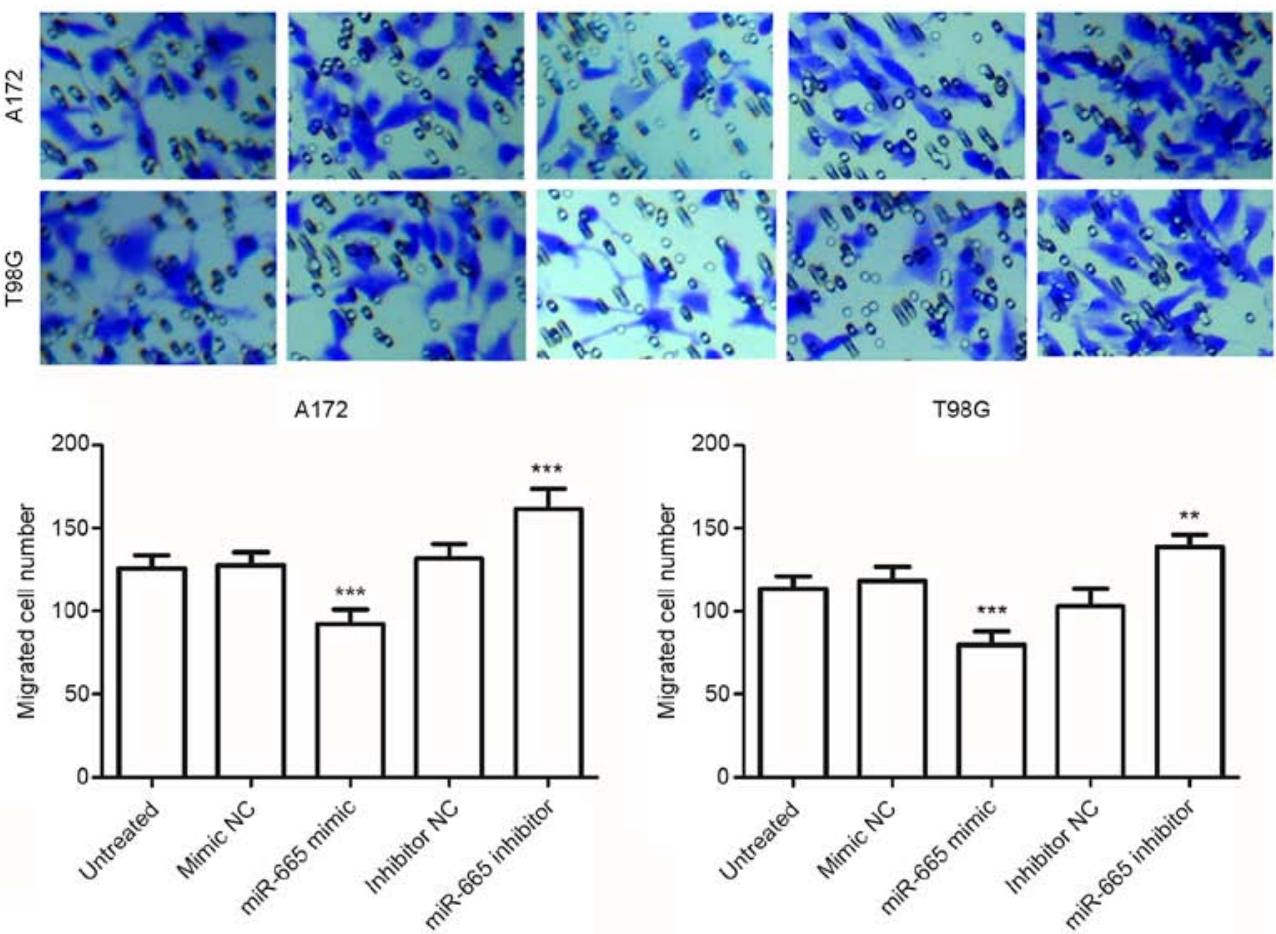

C
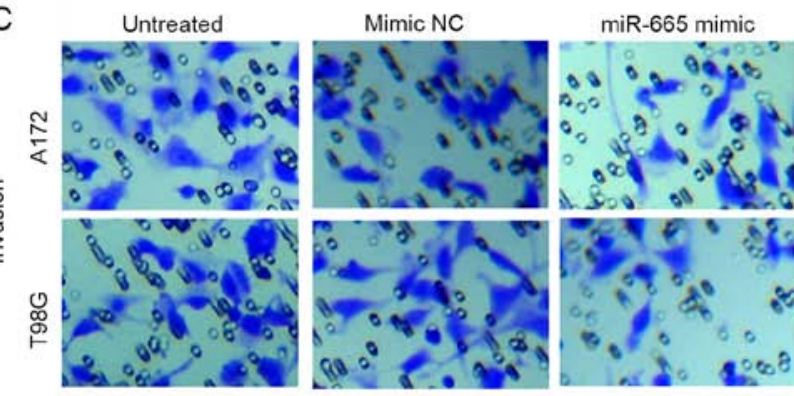

Inhibitor NC miR-665 inhibitor

D

A172
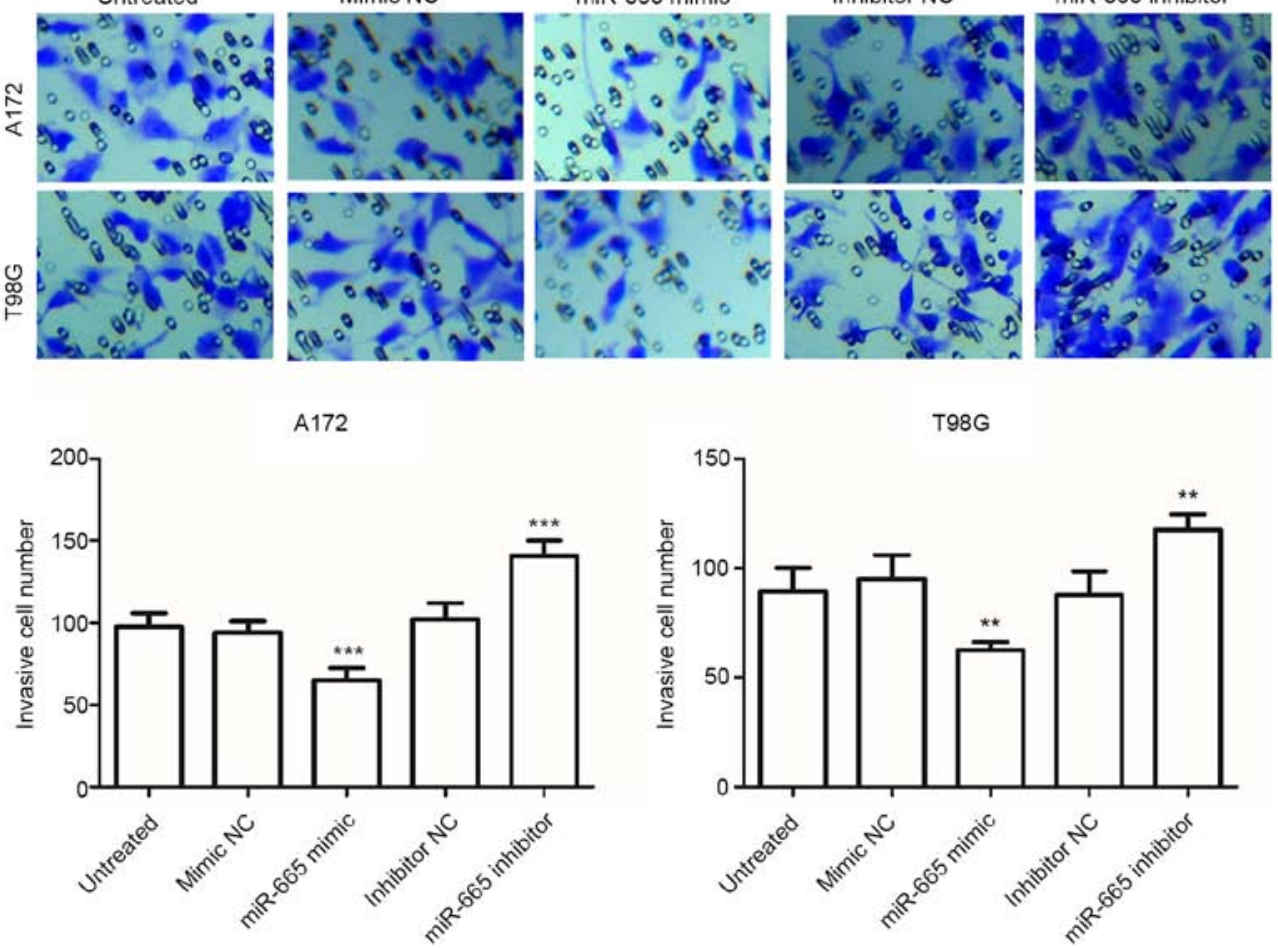

Figure 4. Effect of miR-665 on the migration and invasion of A172 and T98G cells using Transwell migration and invasion assays. (A) Representative migration images of A172 and T98G cells (magnification, x200). (B) Upregulation of miR-665 inhibited cell migration. (C) Representative invasion images of A172 and T98G cells (magnification, $\mathrm{x} 200$ ). (D) Upregulation of miR-665 inhibited invasion, while inhibition of miR-665 enhanced cell migration, compared with untreated cells. ${ }^{* *} \mathrm{P}<0.01$ and ${ }^{* * * *} \mathrm{P}<0.001$ vs. untreated cells. miR, microRNA; NC, negative control.

in certain patients with cancer, such as colon cancer and hepatocellular carcinoma (31,32). In glioma, studies have indicated aberrantly expressed miRNAs are correlated with clinical progression and patient survival, such as miR-3653 (33), miR-362-3p (34) and miR-526b-3p (35). The present study focused on the clinical significance and function of miR-665.
In the present study, miR-665 was significantly downregulated in glioma tissues compared with normal tissues, as well as in glioma cells compared with NHAs. Besides, downregulation of miR-665 was associated with KPS and WHO grade, which suggested that miR-665 acts as a negative regulator in the progression of glioma. Furthermore, the Kaplan-Meier survival 
A

Position 1686-1692 of HMGB1 3'-UTR $55^{\prime} \quad$...UACUGAACAUCUGAGUCCUGgAU...

hsa-miR-665

$3^{\prime}$

|| | | ||

B
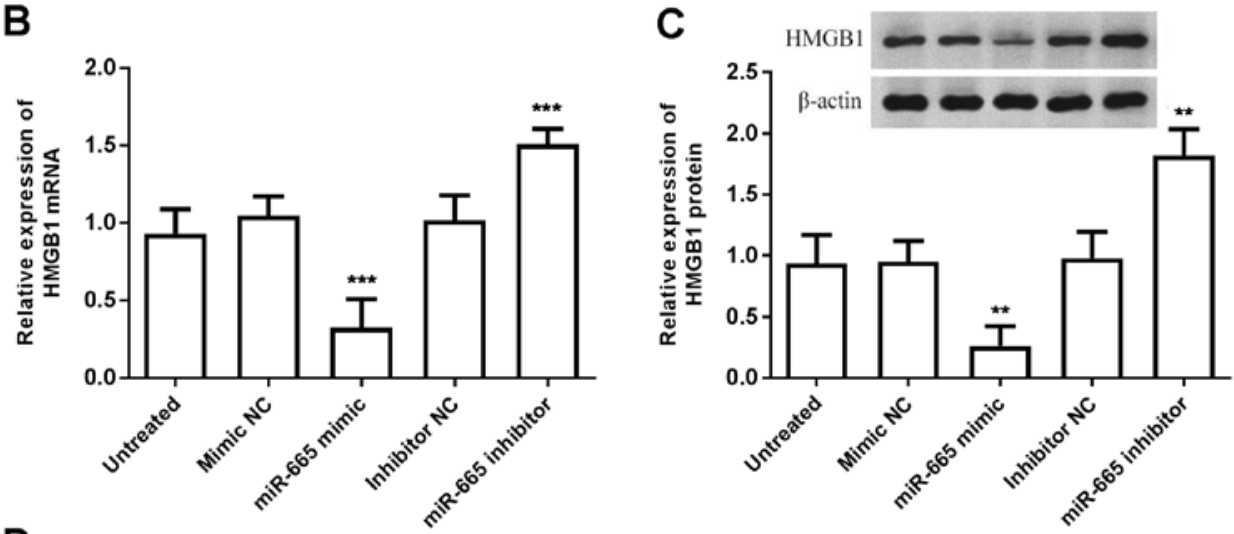

D

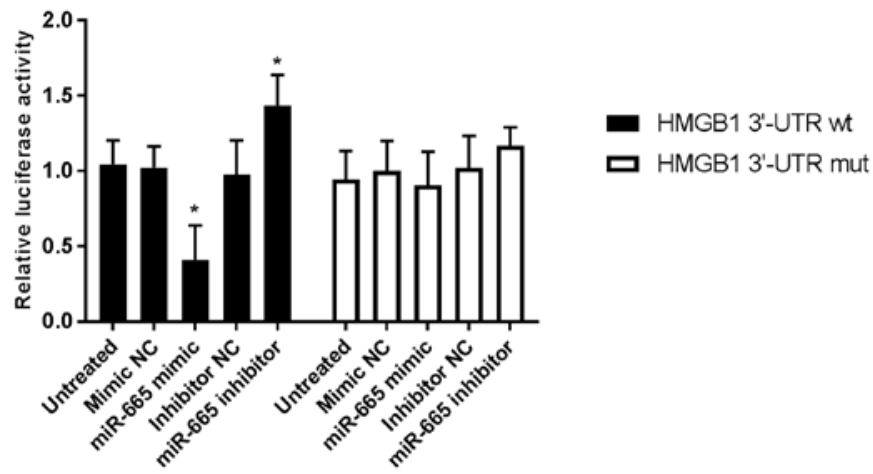

Figure 5. $H M G B 1$ is a target of miR-665. (A) $H M G B 1$ predicted as the target of miR-665 using TargetScan and miRWalk. (B) Overexpression of miR-665 in A172 cells inhibited the expression of $H M G B 1$ mRNA levels. (C) Upregulation of miR-665 led to decreased expression of HMGB1 protein levels. (D) Luciferase activity of the HMGB1 3'-UTR wt was decreased by the miR-665 mimic but increased by the miR-665 inhibitor. ${ }^{*} \mathrm{P}<0.05,{ }^{* * *} \mathrm{P}<0.01$ and ${ }^{* * *} \mathrm{P}<0.001$ vs. untreated cells. HMGB1, high motility box group 1; hsa, homosapien; miR, microRNA; 3'-UTR, 3'-untranslated region; NC, negative control; wt, wild-type; mut, mutant.
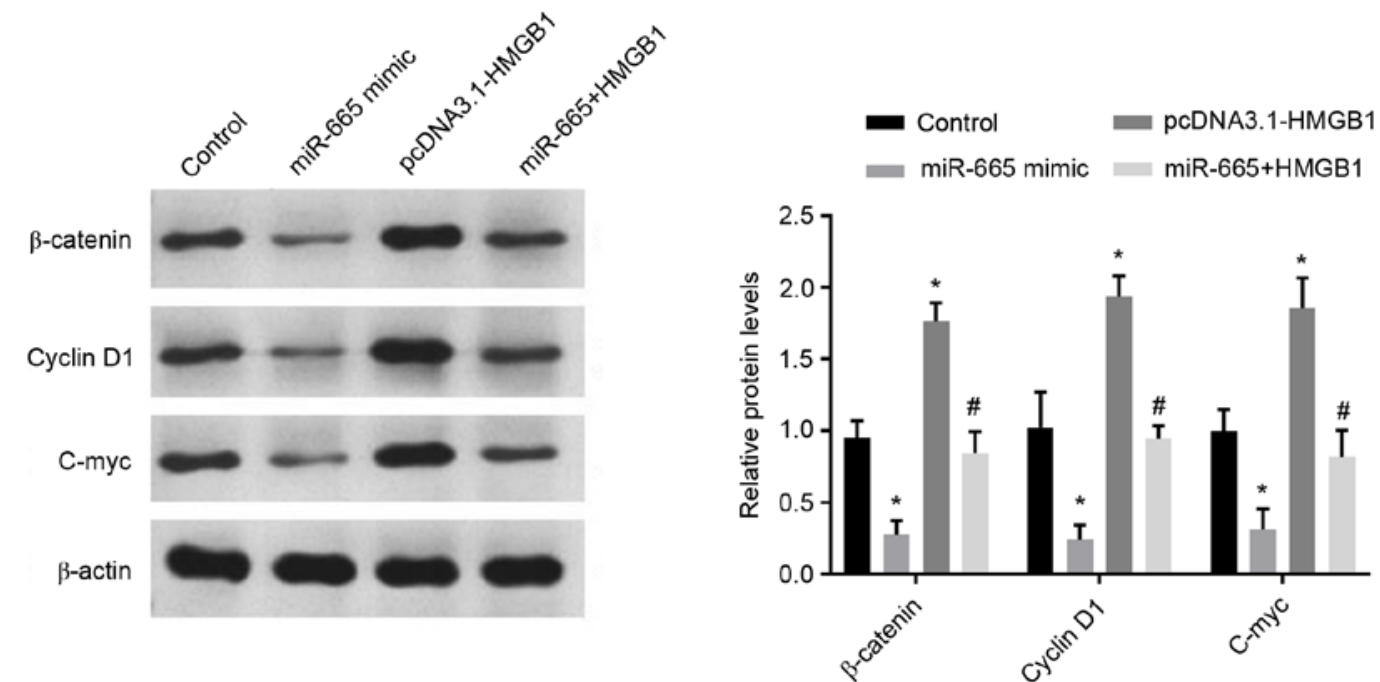

Figure 6. Effects of miR-665 and $H M G B 1$ on the activity of the Wnt/ $\beta$-catenin pathway. Relative protein levels of $\beta$-catenin, cyclin D1 and C-myc were measured using western blotting. " $\mathrm{P}<0.05$ vs. control, ${ }^{\text {P }}<0.05$ vs. pcDNA3.1-HMGB1. HMGB1, high motility box group 1 ; miR, microRNA.

analyses results showed that patients with low expression levels of miR-665 had a shorter overall survival time compared with those with high expression of miR-665. Of note, the multivariate analysis confirmed that miR-665 was an independent predictor of poor prognosis, suggesting that miR-665 may be a novel prognostic biomarker for patients with glioma.

Recent studies have shown that miRNA can exert an oncogenic or suppressor role in human cancer $(36,37)$. For 
instance, miR-6852 expression is downregulated in glioma and suppresses cell proliferation, migration and invasion by targeting lymphoid enhancer-binding factor 1 (38). miR-454-3p exerts tumor-suppressive functions in glioblastoma, inhibiting cell proliferation and inducing apoptosis by targeting nuclear factor of activated T-cells, cytoplasmic 2 (39). Several studies have reported that miR-mediates disease progression in various types of cancer. For instance, Zhao et al (40) demonstrated that miR-665 expression is upregulated and associated with poor survival in patients with breast cancer, and promotes the progression of breast cancer by targeting nuclear receptor subfamily 4 group A member 3 . Hu et al (41) reported that miR-665 expression is increased in hepatocellular carcinoma tissues and cells, and promotes tumor proliferation, migration and invasion, suggesting that miR-665 is a potential target for hepatocellular carcinoma treatment. On the other hand, the suppressor effects of miR-665 have also been observed in several other types of cancer, such as retinoblastoma (28), cervical (42) and ovarian cancer (43). In ovarian cancer, miR-665 suppresses the proliferation and migration of ovarian cancer cells by targeting homeobox protein Hox-A10, which may act as a tumor-suppressing gene (43). These studies suggest that miR-665 may exert an oncogenic or suppressor role in cancer, depending on the cancer type.

In the present study, overexpression of miR-665 inhibited cell proliferation, migration and invasion of glioma cells, while the opposite effects were observed following knockdown of miR-665. Taken together, the data suggested that miR-665 functioned as a tumor suppressor in glioma, which was consistent with the effects of miR-665 in previous studies $(28,42,43)$. In retinoblastoma, miR-665 is markedly reduced and has as a tumor-suppressive role by targeting HMGB1, suggesting that miR-665 is a candidate prognostic biomarker and therapeutic target for patients with retinoblastoma (28). The clinical role and oncogenic function role of HMGB1 have been reported in non-small cell lung cancer $(44)$ and gastric cancer $(45,46)$. In glioma, HMGB1 mediates tumor progression through promoting tumor cell proliferation and the invasion of glioma cells $(47,48)$. In the present study, HMGB1 was predicted as a direct target of miR-665. Furthermore, the present study also focused on the effects of miR-665 and HMGB1 on the activity of the Wnt $/ \beta$-catenin pathway, which contributed to tumor progression. The results revealed that miR-665 inactivated the Wnt/ $\beta$-catenin signaling pathway by targeting HMGB1 in A172 cells.

However, there are still some limitiations in the present study. The design and fieldwork was conducted before publication of the study, thus, the primary limitiation may be the limited sample size. Besides, there are lack of some experimental studies, such as in vivo studies. In the future studies, more experimental verfication with large sample sizes are necessary to understand the molecular mechanism of miR-665 in glioma.

Taken together, the present study demonstrated that miR-665 was downregulated in glioma tissues and cell lines, and predicted poor prognosis of patients with glioma. Besides, miR-665 inhibited the proliferation, migration and invasion of glioma cells by targeting HMGB1 and thereby inactivating the Wnt/ $\beta$-catenin signaling pathway. The present study suggested that miR-665 may be a novel prognostic biomarker and the miR-665/HMGB1 axis may provide a potential therapeutic target for glioma.

\section{Acknowledgements}

Not applicable.

\section{Funding}

No funding was received.

\section{Availability of data and materials}

The datasets used and/or analyzed during the current study are available from the corresponding author on reasonable request.

\section{Authors' contributions}

HS and MX conceived and designed the experiments and contributed significantly to the analysis and manuscript preparation. HS, LX, HT and CY performed the experiments. HS, LX and $\mathrm{HW}$ performed the statistical analysis. HW and YZ the collected data and interpreted the results. All authors read and approved the final manuscript.

\section{Ethics approval and consent to participate}

The present study was approved by The Ethics Committee of Affiliated Hospital of Zunyi Medical University (approval no. SYXK Qin 2018-0006), and written informed consent was obtained from the patients with glioma.

\section{Patient consent for publication}

Not applicable.

\section{Competing interests}

The authors declare that they have no competing interests.

\section{References}

1. Louis DN, Perry A, Reifenberger G, von Deimling A, Figarella-Branger D, Cavenee WK, Ohgaki H, Wiestler OD, Kleihues P and Ellison DW: The 2016 World Health Organization Classification of tumors of the central nervous system: A summary. Acta Neuropathol 131: 803-820, 2016.

2. Hummel S, Kohlmann W, Kollmeyer TM, Jenkins R, Sonnen J, Palmer CA, Colman H, Abbott D, Cannon-Albright L and Cohen AL: The contribution of the rs55705857 G allele to familial cancer risk as estimated in the Utah population database. BMC Cancer 19: 190, 2019.

3. Iser IC, Pereira MB, Lenz G and Wink MR: The epithelial-to-mesenchymal transition-like process in glioblastoma: An updated systematic review and in silico investigation. Med Res Rev 37: 271-313, 2017.

4. Jiang T, Mao Y, Ma W, Mao Q, You Y, Yang X, Jiang C, Kang C, Li X, Chen L, et al: CGCG clinical practice guidelines for the management of adult diffuse gliomas. Cancer Lett 375: 263-273, 2016.

5. Champ CE, Palmer JD, Volek JS, Werner-Wasik M, Andrews DW, Evans JJ, Glass J, Kim L and Shi W: Targeting metabolism with a ketogenic diet during the treatment of glioblastoma multiforme. J Neurooncol 117: 125-131, 2014.

6. Linz U: Commentary on Effects of radiotherapy with concomitant and adjuvant temozolomide versus radiotherapy alone on survival in glioblastoma in a randomised phase III study: 5-year analysis of the EORTC-NCIC trial (Lancet Oncol. 2009;10:459-466). Cancer 116: 1844-1846, 2010.

7. Arvold ND and Reardon DA: Treatment options and outcomes for glioblastoma in the elderly patient. Clin Interv Aging 9: 357-367, 2014. 
8. Hammond SM: An overview of microRNAs. Adv Drug Deliv Rev 87: 3-14, 2015.

9. Kim DY and Sung JH: Regulatory role of microRNAs in the proliferation and differentiation of adipose-derived stem cells Histol Histopathol 32: 1-10, 2017.

10. Mahmoudian-Sani MR, Jalali A, Jamshidi M, Moridi H, Alghasi A, Shojaeian A and Mobini GR: Long non-coding RNAs in thyroid cancer: Implications for pathogenesis, diagnosis, and therapy. Oncol Res Treat 42: 136-142, 2019.

11. Rivera-Barahona A, Pérez B, Richard E and Desviat LR: Role of miRNAs in human disease and inborn errors of metabolism. J Inherit Metab Dis 40: 471-480, 2017.

12. Paliouras AR, Monteverde T and Garofalo M: Oncogene-induced regulation of microRNA expression: Implications for cancer initiation, progression and therapy. Cancer Lett 421: 152-160, 2018.

13. Wu Y, Song Y, Xiong Y, Wang X, Xu K, Han B, Bai Y, Li L, Zhang Y and Zhou L: MicroRNA-21 (Mir-21) Promotes cell growth and invasion by repressing tumor suppressor PTEN in colorectal cancer. Cell Physiol Biochem 43: 945-958, 2017.

14. Liu DZ, Zhao H, Zou QG and Ma QJ: MiR-338 suppresses cell proliferation and invasion by targeting CTBP2 in glioma. Cancer Biomark 20: 289-297, 2017.

15. Svoronos AA, Engelman DM and Slack FJ: OncomiR or tumor suppressor? The duplicity of MicroRNAs in cancer. Cancer Res 76: 3666-3670, 2016

16. Möhnle P, Schütz SV, Schmidt M, Hinske C, Hübner M, Heyn J, Beiras-Fernandez A and Kreth S: MicroRNA-665 is involved in the regulation of the expression of the cardioprotective cannabinoid receptor $\mathrm{CB} 2$ in patients with severe heart failure. Biochem Biophys Res Commun 451: 516-521, 2014

17. Tan H, Zhao L, Song R, Liu Y and Wang L: microRNA-665 promotes the proliferation and matrix degradation of nucleus pulposus through targeting GDF5 in intervertebral disc degeneration. J Cell Biochem 119: 7218-7225, 2018.

18. Liu NW, Huang X, Liu S and Lu Y: EXT1, regulated by MiR-665, promotes cell apoptosis via ERK1/2 signaling pathway in acute lymphoblastic leukemia. Med Sci Monit 25: 6491-6503, 2019.

19. Ye X, Wei W, Zhang Z, He C, Yang R, Zhang J, Wu Z, Huang Q and Jiang Q: Identification of microRNAs associated with glioma diagnosis and prognosis. Oncotarget 8: 26394-26403, 2017.

20. Tesarova P, Kalousova M, Zima T and Tesar V: HMGB1, S100 proteins and other RAGE ligands in cancer-markers, mediators and putative therapeutic targets. Biomed Pap Med Fac Univ Palacky Olomouc Czech Repub 160: 1-10, 2016.

21. Wang Y, Jiang Z, Yan J and Ying S: HMGB1 as a potential biomarker and therapeutic target for malignant mesothelioma. Dis Markers 2019: 4183157, 2019.

22. Wu T, Zhang W, Yang G, Li H, Chen Q, Song R and Zhao L: HMGB1 overexpression as a prognostic factor for survival in cancer: A meta-analysis and systematic review. Dis Markers 7: 50417-50427, 2016.

23. Zhao Y, Yi J, Tao L, Huang G, Chu X, Song H and Chen L: Wnt signaling induces radioresistance through upregulating HMGB1 in esophageal squamous cell carcinoma. Cell Death Dis 9: 433, 2018.

24. Reed KR, Song F, Young MA, Hassan N, Antoine DJ, Gemici NB, Clarke AR and Jenkins JR: Secreted HMGB1 from Wnt activated intestinal cells is required to maintain a crypt progenitor phenotype. Oncotarget 7: 51665-51673, 2016.

25. Nagane M, Kobayashi K, Ohnishi A, Shimizu S and Shiokawa Y Prognostic significance of O6-methylguanine-DNA methyltransferase protein expression in patients with recurrent glioblastoma treated with temozolomide. Jpn J Clin Oncol 37: 897-906, 2007.

26. Li C, Zou H, Wang Z, Tang X, Fan X, Zhang K, Liu J and Li Z REST, not REST4, is a risk factor associated with radiotherapy plus chemotherapy efficacy in glioma. Drug Des Devel Ther 12 1363-1371, 2018.

27. Livak KJ and Schmittgen TD: Analysis of relative gene expression data using real-time quantitative PCR and the 2(-Delta Delta C(T)) method. Methods 25: 402-408, 2001.

28. Wang S, Du S, Lv Y, Zhang F and Wang W: MicroRNA-665 inhibits the oncogenicity of retinoblastoma by directly targeting high-mobility group box 1 and inactivating the Wnt/ $\beta$-catenin pathway. Cancer Manag Res 11: 3111-3123, 2019.
29. Zhang H, Li X, Meng W, Zhang L, Zhu X, Bai Z, Yan J and Zhou W: Overexpression of p16ink4a regulates the Wnt/beta-catenin signaling pathway in pancreatic cancer cells. Mol Med Rep 17: 2614-2618, 2018.

30. Chen W, Zheng R, Baade PD, Zhang S, Zeng H, Bray F, Jemal A, $\mathrm{Yu}$ XQ and He J: Cancer statistics in China, 2015. CA Cancer J Clin 66: 115-132, 2016.

31. Dehghan F, Boozarpour S, Torabizadeh Z and Alijanpour S: miR-21: A promising biomarker for the early detection of colon cancer. Onco Targets Ther 12: 5601-5607, 2019.

32. Ning S, Liu H, Gao B, Wei W, Yang A, Li J and Zhang L: miR-155, miR-96 and miR-99a as potential diagnostic and prognostic tools for the clinical management of hepatocellular carcinoma. Oncol Lett 18: 3381-3387, 2019.

33. Chen Y, Li ZH, Liu X, Liu GX, Yang HM and Wu PF: Reduced expression of miR-3653 in glioma and its correlations with clinical progression and patient survival. Eur Rev Med Pharmacol Sci 23: 6596-6601, 2019.

34. Xu G, Fang P, Chen $\mathrm{K}, \mathrm{Xu} \mathrm{Q}$, Song $\mathrm{Z}$ and Ouyang $\mathrm{Z}$ MicroRNA-362-3p Targets PAX3 to Inhibit the Development of Glioma through Mediating Wnt/beta-Catenin Pathway. Neuroimmunomodulation 26: 119-128, 2019.

35. Wu M, Li X, Liu Q, Xie Y, Yuan J and Wanggou S: miR-526b-3p serves as a prognostic factor and regulates the proliferation, invasion, and migration of glioma through targeting WEE1. Cancer Manag Res 11: 3099-3110, 2019.

36. Sun X, Cui S, Fu X, Liu C, Wang Z and Liu Y: MicroRNA-146-5p promotes proliferation, migration and invasion in lung cancer cells by targeting claudin-12. Cancer Biomark 25: 89-99, 2019.

37. Chen S, Shi F, Zhang W, Zhou Y and Huang J: miR-744-5p inhibits non-small Cell lung cancer proliferation and invasion by directly targeting PAX2. Technol Cancer Res Treat 18: $1533033819876913,2019$.

38. Wang J, Liu H, Zheng K, Zhang S and Dong W: MicroRNA-6852 suppresses glioma A172 cell proliferation and invasion by targeting LEF1. Exp Ther Med 18: 1877-1883, 2019.

39. Zuo J, Yu H, Xie P, Liu W, Wang K and Ni H: miR-454-3p exerts tumor-suppressive functions by down-regulation of NFATc2 in glioblastoma. Gene 710: 233-239, 2019.

40. Zhao XG, Hu JY, Tang J, Yi W, Zhang MY, Deng R, Mai SJ, Weng NQ, Wang RQ, Liu J, et al: miR-665 expression predicts poor survival and promotes tumor metastasis by targeting NR4A3 in breast cancer. Cell Death Dis 10: 479, 2019.

41. Hu Y, Yang C, Yang S, Cheng F, Rao J and Wang X: miR-665 promotes hepatocellular carcinoma cell migration, invasion, and proliferation by decreasing Hippo signaling through targeting PTPRB. Cell Death Dis 9: 954, 2018.

42. Cao L, Jin H, Zheng Y, Mao Y, Fu Z, Li X and Dong L: DANCR-mediated microRNA-665 regulates proliferation and metastasis of cervical cancer through the ERK/SMAD pathway. Cancer Sci 110: 913-925, 2019.

43. Liu J, Jiang Y, Wan Y, Zhou S, Thapa S and Cheng W: MicroRNA665 suppresses the growth and migration of ovarian cancer cells by targeting HOXA10. Mol Med Rep 18: 2661-2668, 2018.

44. Ma Y, Kang S, Wu X, Han B, Jin Z and Guo Z: Up-regulated HMGB1 in the pleural effusion of non-small cell lung cancer (NSCLC) patients reduces the chemosensitivity of NSCLC cells Tumori 104: 338-343, 2018.

45. Li Y and Qin C: MiR-1179 inhibits the proliferation of gastric cancer cells by targeting HMGB1. Hum Cell 32: 352-359, 2019.

46. Zhang J, Zhang R, Lu WW, Zhu JS, Xia LQ, Lu YM and Chen NW: Clinical significance of hmgbl expression in human gastric cancer. Int J Immunopathol Pharmacol 27: 543-551, 2014.

47. Angelopoulou E, Piperi C, Adamopoulos C and Papavassiliou AG: Pivotal role of high-mobility group box 1 (HMGB1) signaling pathways in glioma development and progression. J Mol Med (Berl) 94: 867-874, 2016.

48. Zhang J, Liu C and Hou R: Knockdown of HMGB1 improves apoptosis and suppresses proliferation and invasion of glioma cells. Chin J Cancer Res 26: 658-668, 2014. 